\title{
Inhibition of TGF $\beta$-induced Fibroblast to Myofibroblast Transition by Conditioned Medium From Human Rhinovirus-infected Airway Epithelial Cells is Mediated by Prostaglandin $\mathrm{E}_{2}$
}

\author{
Diana Pham \\ University of Calgary \\ Cora Kooi \\ University of Calgary \\ David Proud \\ University of Calgary
}

Richard Leigh ( $\sim$ rleigh@ucalgary.ca )

University of Calgary

\section{Research Article}

Keywords: Human rhinovirus, epithelial cells, fibroblasts, myofibroblasts, prostanoids

Posted Date: February 4th, 2022

DOI: https://doi.org/10.21203/rs.3.rs-1296940/v1

License: (a) (i) This work is licensed under a Creative Commons Attribution 4.0 International License.

Read Full License 


\section{Abstract \\ Background}

Airway remodeling, including increased deposition of extracellular matrix proteins, is a hallmark of asthma. Myofibroblasts, derived via fibroblast to myofibroblast transition (FMT), play an important role in this increased deposition of matrix proteins. Expression of transforming growth factor $\beta 1$ (TGF $\beta 1$ ) is increased in asthmatic airways and is known to induce FMT, as assessed by increased expression of asmooth muscle actin (a-SMA). Because human rhinovirus (HRV) infections of airway epithelial cells have been linked to the generation of mediators involved in airway remodeling, we tested the hypothesis that conditioned medium from HRV-infected human airway epithelial cells from healthy normal subjects would enhance FMT, either alone or in combination with TGF $\beta 1$.

\section{Methods}

FMT was assessed by measuring a-SMA expression in human airway fibroblasts, both by western blotting and by immunohistochemistry. Statistical analysis was performed using ANOVA and Holm-Sidak or Bonferonni post hoc tests.

\section{Results}

Our data showed that conditioned medium from HRV infected epithelial cells significantly inhibited TGF $\beta 1$-induced expression of a-SMA in fibroblasts when compared to conditioned medium from uninfected epithelial cells. Treatment of epithelial cells with the nonselective cyclooxygenase inhibitor, diclofenac, during HRV infection significantly reversed the inhibitory effects of conditioned medium on TGF $\beta 1$-induced expression of a-SMA. This led us to hypothesize that HRV-infected epithelial cells release $\mathrm{PGE}_{2}$ that acts on EP receptors to suppress TGF $\beta 1$-induced expression of a-SMA in fibroblasts. We confirmed that $\mathrm{HRV}$-infection of airway epithelial cells upregulated $\mathrm{PGE}_{2}$ release, and that exposure of fibroblasts to $\mathrm{PGE}_{2}$ significantly inhibited TGF $\beta 1$-induced expression of a-SMA in fibroblasts. Finally, studies using selective agonists and antagonists demonstrated that $\mathrm{PGE}_{2}$ inhibited TGF $\beta 1$-induced expression of a-SMA in fibroblasts via effects at the $\mathrm{EP}_{2}$ receptor.

\section{Conclusions}

Thus, using cells derived from healthy normal subjects we demonstrate that HRV infection of airway epithelial cells induces release of $\mathrm{PGE}_{2}$ that acts on $\mathrm{EP}_{2}$ receptors on fibroblasts to suppress TGF $\beta 1$ induced FMT. Additional studies are needed to determine if a different pattern of response is seen using cells from asthmatic subjects to determine if the inhibitory role of $\mathrm{PGE}_{2}$ on FMT in normal cells helps prevent airway remodeling in normal subjects. 


\section{Introduction}

The term airway remodeling is used to describe the collection of structural changes in the airways of patients with asthma. These changes, which begin to manifest in early childhood, include increased mass of airway smooth muscle, goblet cells hypertrophy and associated mucus hypersecretion, angiogenesis, and increased matrix protein deposition, particularly in the lamina reticularis [1, 2]. Increased matrix protein deposition is associated with increased numbers of fibroblasts and myofibroblasts near the laminar reticularis [1,3]. Both fibroblasts and myofibroblasts can secrete multiple extracellular matrix proteins. Myofibroblasts, display characteristics of both fibroblasts and smooth muscle cells [4], making them difficult to identify, except via ultrastructural analysis using electron microscopy, which is considered the "gold standard" for identifying these cells [5, 6]. Myofibroblasts, which, unlike fibroblasts, express a-smooth muscle actin (a-SMA) [7] are thought to be more metabolically active than fibroblasts based on greater expression and enhanced production of matrix proteins, including collagens I, III and V, as well as fibronectin and tenascin $[8,9]$. As such, they are thought to be important contributors to remodeling in asthma [10].

The origin of myofibroblasts is a topic of debate with suggestions that they can derive from fibrocytes, via epithelial mesenchymal transition, or from smooth muscle to myofibroblast transition [11-13]. Considerable evidence, however, also supports that fibroblast to myofibroblast transition (FMT) is a source of myofibroblasts in airway tissues [8]. This process, which includes increased expression of aSMA, activation of focal adhesion kinases, and formation of stress fibers ultimately leads to myofibroblasts that exhibit decreased motility and proliferative abilities but increased contractile properties and enhanced secretion of matrix proteins $[6,8]$. Various stages of FMT can be regulated by a number of mechanical factors, as well as by cytokines, and growth factors, with transforming growth factor- $\beta$ (TGF $\beta$ ) being the most studied $[13,14]$. It is established that TGF $\beta$, levels of which are increased in asthma [14], can induce FMT in fibroblasts from both healthy and asthmatic subjects with asthmatic cells showing an increased potential to transition $[8,15]$.

Recent studies support the potential for human rhinovirus (HRV) infections to contribute to aspects of airway remodeling by changing the biology of infected airway epithelial cells, one of the initiating cells involved in remodeling [2]. It has been shown that HRV-infected airway epithelial cells release increased levels of several chemokines, growth factors and proteases linked to angiogenesis or to matrix remodeling [16-19]. In addition, there is evidence that HRV infection of airway epithelial cells can contribute to epithelial mesenchymal transition [20]. In the current study, therefore, we examined the potential of HRV infection of human airway epithelial cells to cause the release of products that may regulate FMT either alone, or in combination with TGF $\beta$. Surprising, we demonstrate that conditioned medium from HRV-infected airway epithelial cells significantly inhibits TGF $\beta$-induced FMT via a $\mathrm{PGE}_{2}{ }^{-}$ mediated mechanism.

\section{Methods}




\section{Cell culture}

All tissue culture products were purchased from ThermoFisher Scientific (Waltham, MA) except where indicated. Primary human bronchial epithelial (HBE) and fibroblast (HBF) cells were isolated from nontransplanted normal human lungs obtained through a tissue retrieval service (International Institute for the Advancement of Medicine, Edison, NJ) with approval from the Conjoint Health Research Ethics Board of the University of Calgary and the Internal Ethics Board of the International Institute for the Advancement of Medicine. Airways up to around the 4th generation were dissected from the lung parenchyma. For fibroblast cell isolation, a section of mainstem bronchus was cut up into 1-2 mm pieces and placed in 24-well plates containing dMEM, 10\% fetal bovine serum (FBS) (Invitrogen, Carlsbad, CA). After approximately two weeks, fibroblast cells that migrated out of the tissue plugs were expanded, enumerated and frozen in aliquots at $-80^{\circ} \mathrm{C}$. For epithelial cell isolation, the remaining airway pieces were submerged in sterile Ham's F12 medium containing $1 \mu \mathrm{l} / \mathrm{ml}$ gentamicin and $1 \mathrm{mg} / \mathrm{ml}$ pronase (MilliporeSigma, Burlington, MA) for approximately 36 hours at $4^{\circ} \mathrm{C}$. The airway pieces were then submerged in F12 containing 10\% heat inactivated FBS and epithelial cells were removed from the surface by jetting pre-warmed medium repeatedly over the exposed surface using a $5 \mathrm{ml}$ syringe. Epithelial cells were routinely grown in 6-well plates in complete PromoCell (Heidelberg, Germany) epithelial cell growth medium at $37^{\circ} \mathrm{C}$ for 14 days. Fibroblasts were seeded in either six-well plates or chamber slides at a seeding density of $1.5 \times 10^{6}$ or $0.5 \times 10^{5}$ cells, respectively. HBF cells were grown in dMEM supplemented with $10 \%$ fetal bovine serum (FBS). For consistency, conditioned medium from 6 different HBE donors were pooled and aliquoted to use in experiments. Values of $\mathrm{n}$ correspond to the number of different individual fibroblast donors used in each experiment. Information on HBE and HBF donors used is provided (Supplemental Table 1).

\section{HRV purification}

HRV major group strain 16 (HRV-16) was propagated in WI-38 fetal lung fibroblasts cells (American Type Culture Collection, Rockville, MD) and purified by sucrose density centrifugation as previously described [21].

\section{Treatment of human bronchial epithelial cells}

After 14 days of growth, HBE cells at approximately $70 \%$ confluency were pre-treated for $24 \mathrm{~h}$ with PromoCell epithelial growth medium with the hydrocortisone removed to minimize the potential effects of steroids. Four hours prior to treatment, HBE cells were pre-treated with PromoCell basal epithelial medium. HBE cells were infected with HRV-16 at a multiplicity of infection of $\sim 1$ in PromoCell basal medium or treated with basal medium alone. In some experiments, HBE cells were pretreated for 30 min with diclofenac $\left(10^{-6} \mathrm{M}\right)$. The $24 \mathrm{~h}$ conditioned medium was collected and centrifuged at $1200 \mathrm{rpm}$ at $4^{\circ} \mathrm{C}$ for 8 minutes. The supernatant layer was transferred to another tube and stored in aliquots at $-80^{\circ} \mathrm{C}$ for future supernatant analysis or co-culture with fibroblasts.

\section{Enzyme linked immunosorbent assays (ELISAs)}


Levels of CXCL10, CXCL8 and PGE 2 released from the HBE cells in response to viral infection were measured by ELISA. The assay for $\mathrm{PGE}_{2}$ was purchased from Cayman Chemical (Ann Arbor, MI). CXCL10 was assayed using antibodies from Peprotech (East Windsor, NJ). Levels of CXCL8 were measured using a previously described ELISA developed in our laboratory [22].

\section{Treatment of human bronchial fibroblast cells}

HBF cells were pre-treated with dMEM, PSF and no serum for 2 hours prior to exposure. Fibroblasts were then treated with a 1:1 ( $\mathrm{vol} / \mathrm{vol})$ mixture of dMEM containing $0.1 \%$ BSA and conditioned medium from either HBE infected with HRV or uninfected control HBE. TGF 1 1 was also added with certain treatments to act as a positive control or as a co-stimulus. In some experiments HBF cells were pre-treated with individual antagonists for the $\mathrm{EP}_{1}$ receptor (SC51322), $\mathrm{EP}_{2}$ receptor (TG4-155), $\mathrm{EP}_{3}$ receptor (L798106), $\mathrm{EP}_{3}$ antagonist (L798106), or the $\mathrm{EP}_{4}$ receptor (L161982). Cells were preincubated with selective $\mathrm{EP}$ receptor antagonists $(100 \mathrm{mM})$ for $30 \mathrm{~min}$. For these experiments, each antagonist was also present throughout the $48 \mathrm{~h}$ incubation. In other experiments, HBF cells were treated with the $\mathrm{EP}_{1-4}$ agonists, ONOD1-004, ONO-AE1-259-01, ONO-AE-248, ONO-AE1-329, repectively (generously provided by Dr. Mark Giembycz, University of Calgary). After 48 hours, a-SMA expression levels were assessed by immunochemistry and/or western blotting. Cell viability was also assessed using a Thiazolyl Blue Tetrazolium Bromide (MTT) assay (MilliporeSigma).

\section{Immunohistochemical assessment of a-SMA expression in fibroblasts}

After 48 hours of treatment, chamber slides containing HBF cells were fixed in a $25 \%$ acetone/ $75 \%$ ethanol mixture for 10 minutes at $-20^{\circ} \mathrm{C}$. The slides were treated with $0.03 \% \mathrm{H}_{2} \mathrm{O}_{2}$ in phosphate buffered saline (PBS) for 3 minutes. Following three PBS washes ( $\mathrm{pH} 7.2-7.6$ ), the cells were blocked with a $2 \%$ normal goat serum application for 30 minutes. a-SMA primary antibody (catalog \# ab5694; Abcam, Cambridge, UK) diluted 1:125 in antibody diluent (DAKO, Santa Clara, CA) was applied for 90 minutes at room temperature. Following thorough PBS washing, a 1:200 dilution of secondary antibody in antibody diluent was applied for 45 minutes at room temperature. After washing with PBS, the slides were incubated with peroxidase labelled avidin biotin complex for 30 minutes at room temperature. Washes with PBS and rinses with distilled $\mathrm{H}_{2} \mathrm{O}$ were then performed prior to a 5 minute application of 3,3'Diaminobenzidine (DAB) chromogen solution. The slides were washed five times with $\mathrm{dH} 2 \mathrm{O}$ and then counterstained with Gills II hematoxylin for 20 seconds followed by warm water for 2 minutes. A series of dehydration steps was then performed using $95 \%$ ethanol and 2 changes of $100 \%$ isopropanol for 2 minutes at each change, followed by 2 changes in xylenes for 2 minutes before being mounted in permount (Thermofisher) for cover slip attachment. Chamber slides were left to dry for at least 24 hours before microscopic analysis.

\section{Quantification of a-SMA positive cells by immunohistochemistry}


A cell was considered a-SMA positive if they presented with a Hematoxylin-stained nucleus with brownstained striations (a-SMA). TGF $\beta 1$ alone in media was used as a positive control on fibroblasts as it is well-established to induce a-SMA expression [15]. To determine the average amount of a-SMA positive cells per condition, a-SMA positive cells in the field of vision at 100X magnification at each of the four corners and the center of the slide were counted and summed together. The total number a-SMA positive cells counted were then divided by 5 as there were a total of five fields of vision; thus, representing the average number of a-SMA positive cells on the slide.

\section{Whole-cell lysates}

Post treatment, supernatants were aspirated, cells were washed with HBSS and fibroblasts were lysed in ice-cold lysis cocktail buffer made of $0.81 \%$ Triton X-100 in 1 X MES buffered saline pH 7.4 containing 2 $\mathrm{mM}$ sodium orthovandate, $20 \mathrm{mM}$ sodium pyrophosphate, $50 \mathrm{mM}$ sodium fluoride, $1 \mathrm{X}$ complete protease inhibitor (Roche, Basel, Switzerland) and $1 \mathrm{mM}$ of phenylmethylsulfonyl fluoride (PMSF). To further ensure complete cell lysis, the cells in lysis buffer were placed at $-80 \mathrm{C}$ to undergo a freeze-thaw cycle, scraped and sonicated using a Branson 2510 Sonicator for 30 seconds. After sonication, the lysates were centrifuged at $10,000 \mathrm{xg}$ for $5 \mathrm{~min}$ and the lysate supernatants were transferred to clean tubes. Lysates were analyzed for protein content using a DC Protein Assay (BioRad Laboratories, Mississauga, ON, Canada) as recommended by the manufacturer.

\section{Western blots}

Equivalent amounts of whole cell lysate were separated by $12 \%$ Sodium Dodecyl Sulfate-Polyacrlamide Gel Electrophoresis (SDS-PAGE), and then transferred to a $0.45 \mu \mathrm{m}$ nitrocellulose membrane. Membranes were blocked with $3 \%$ bovine serum albumin (BSA) in $0.05 \%$ Tween 20 tris-buffered saline (TTBS) for $1 \mathrm{~h}$. The blots were washed three times in $0.05 \%$ TTBS for at least 5 min each time, followed by transfer into roller tubes containing a-SMA primary antibody in 3\% BSA in 0.05\% TTBS at a 1:2000 dilution, and incubated overnight at $4^{\circ} \mathrm{C}$ on a roller mixer. Following three 15 min washes, the blots were incubated with a-rabbit Ig-horseradish peroxidase (HRP) secondary antibody at a 1:10000 dilution in $0.1 \%$ TTBS for 1 hour followed by three washes with $0.05 \%$ TTBS for 15 minutes. a-SMA protein levels were visualized using a digital imager (Bio-Rad, Hercules, $\mathrm{CA}$ ) after a 1 min exposure to enhanced chemiluminescence $(E C L)$ reagent for visualization of HRP activity. Membranes were then stripped and re-probed using antibody (AbD Serotec, Raleigh NC) to the housekeeping protein, glyceraldehyde 3-phosphate dehydrogenase (GAPDH).

\section{Densitometry analysis}

Densitometry analysis was performed using ImageJ software (version 1.41, National Institute of Health, Bethesda, MD). The fold change in expression of the protein of interest was determined by normalizing the raw pixel count of the protein of interest to the raw pixel count of the housekeeping protein, GAPDH. This was then compared to the normalized value for medium control. Data are expressed as fold change over medium control unless specified otherwise. 


\section{$E P_{1-4}$ receptor expression in HBF cells}

To examine mRNA expression of PGE2 receptors in HBF, total cellular RNA was purified from cells using a Nucleospin isolation kit (Macherey-Nagal, Duren, Germany) and converted to cDNA using EasyScript ${ }^{\text {tM }}$ reverse transcriptase (Applied Biological Materials, Inc., Richmond, BC, Canada) as recommended by the manufacturers. $\mathrm{EP}_{1-4}$ receptors (PTGER1-PTGER4) were amplified and quantified using the primer sequences specific to each receptor (Supplemental Table 2). Relative quantification of each EP receptor gene expression was achieved by comparing to a 7-point standard curve generated using cDNA from myometrial cells, which highly express each receptor. GAPDH was used to ensure equal cDNA input.

\section{Statistical analysis}

All statistics were performed using GraphPad Prism 6 (GraphPad Software, San Diego, CA). All data were expressed as mean \pm standard error of the mean. The Kolmogorov-Smirnov normality test determined the parametric or non-parametric status of the data sets. Parametric data were analyzed by one-way ANOVA with appropriate post hoc test. Data sets comparing two conditions were analyzed by using a paired or unpaired student t-test, as appropriate. For all statistical tests, a two-tailed p-value less than 0.05 was considered statistically significant $\left({ }^{\star} p<0.05,{ }^{\star \star} p<0.01,{ }^{\star \star *} p<0.001,{ }^{\star \star \star \star} p<0.0001\right)$.

\section{Results}

We used western blotting and immunohistochemistry the evaluate the effects of conditioned medium from HRV-infected and uninfected (control) HBE on expression of a-SMA in fibroblasts, both alone, and in the presence of TGF $\beta 1$. Compared to conditioned medium from control HBE, conditioned medium from infected HBE caused a consistent, but not statistically significant, reduction in a-SMA expression when used alone. We confirmed prior data that TGF $\beta 1$ enhances fibroblast expression of a-SMA in a dosedependent manner (Supplemental Figure 1). When conditioned medium from infected and non-infected HBE were used in conjunction with TGF $\beta 1$, conditioned medium from HRV-infected HBE significantly decreased expression of a-SMA when compared with control conditioned medium (Figure 1A, B). This reduction was not due to cell death (Supplemental Figure 2). To further validate these observations, we performed a limited number of experiments $(n=3)$ using immunohistochemistry for a-SMA and observed a similar pattern of a-SMA expression with the various treatments (Figure 1C, D).

Prostaglandins are known to have multiple actions in the asthmatic airways [23]. To evaluate if prostanoids may play a role in the effects of conditioned medium from HRV-infected epithelial cells, we performed western blotting studies using the non-selective cyclooxygenase inhibitor, diclofenac. The data demonstrate that exposure of HBE to diclofenac during HRV-infection significantly reversed the inhibitory effect of conditioned medium on TGF $\beta$-induced a-SMA expression in fibroblasts, although inhibition was not complete (Figure 2A, B). 
Given that $\mathrm{PGE}_{2}$ has previously been shown to downregulate TGF $\beta 1$-induced a-SMA expression [24], we hypothesized that $\mathrm{HRV}$-infected $\mathrm{HBE}$ release increased levels of $\mathrm{PGE}_{2}$ that contribute to the inhibitory effects of HRV-infected conditioned medium on TGF $\beta 1$-induced a-SMA expression. In support of this hypothesis, we observed that, while levels of $\mathrm{PGE}_{2}$ were undetectable in conditioned medium from noninfected epithelial cells, HRV-16 treatment of $\mathrm{HBE}$ cells resulted in a significant increase in $\mathrm{PGE}_{2}$ supernatant levels, with a mean value of $73.7 \mathrm{pg} / \mathrm{ml}$. As expected, HRV-16 infected HBE no longer produced $\mathrm{PGE}_{2}$ in the presence of diclofenac (Figure 3).

We initially examined the effects of varying concentrations of $\mathrm{PGE}_{2}$ on basal expression of a-SMA in HBF cells. Although an inhibitory trend was observed, this did not achieve significance (Supplemental Figure 3). In the presence of $3 \mathrm{ng} / \mathrm{ml}$ of TGF $\beta 1$, however, increasing concentrations of $\mathrm{PGE}_{2}$ significantly decreased TGF $\beta 1$-induced a-SMA protein levels in fibroblasts (Figure 4).

$\mathrm{PGE}_{2}$ binds specifically to $\mathrm{EP}_{1-4}$ receptors, which are coupled to various signaling cascades, depending on receptor subtype. As an initial approach to examine potential receptor involvement, levels of mRNA for each receptor were examined by RT-PCR. The $\mathrm{EP}_{2}$ receptor was the predominant receptor expressed in $\mathrm{HBF}$ cells, followed by the $\mathrm{EP}_{4}$ receptor. Trace amounts of $\mathrm{EP}_{3}$ receptor expression were also detected (Supplemental Figure 4).

Selective EP receptor antagonists and agonists were used to further investigate the receptor-mediated pathway by which $\mathrm{PGE}_{2}$ suppresses TGF $\beta 1$-induced a-SMA. When fibroblasts were exposed to TGF $\beta 1$, in the presence or absence of $\mathrm{PGE}_{2}, \mathrm{TGF} \beta 1$ alone increased $\mathrm{a}-\mathrm{SMA}$ expression as expected. This was suppressed when $\mathrm{PGE}_{2}$ was added. In the presence of selective antagonists for each EP receptor subtype, only the $\mathrm{EP}_{2}$ receptor antagonist reversed PGE2-mediated reduction in TGF $\beta 1$-induced a-SMA expression (Figure 5). To further support the premise that $\mathrm{PGE}_{2}$ selectively binds to the $\mathrm{EP}_{2}$ receptor to mediate inhibition of TGF $\beta 1$ induced a-SMA expression, we examined the effects of selective agonists to each EP receptor subtype. Consistent with the data using antagonists, only the $\mathrm{EP}_{2}$ receptor agonist was able to suppress a-SMA expression in the presence of TGF $\beta 1$ (Figure 6).

\section{Discussion}

Given that earlier studies have shown that HRV-infection of human airway epithelial cells releases products that are known to contribute to airway remodeling [16-19], and that HRV-infections synergizes with TGF $\beta 1$ in triggering epithelial to mesenchymal transition [20], we initially hypothesized that conditioned medium from HRV-infected epithelial cells would enhance TGF $\beta 1$-induced FMT, as monitored by increased a-SMA expression. Our data consistently demonstrated, however, that medium from epithelial cells infected with HRV significantly inhibited the ability of TGF $\beta 1$ to induce a-SMA expression in airway fibroblasts, using both western blotting and immunohistochemistry for a-SMA. 
Studies using the non-selective cyclooxygenase inhibitor, diclofenac, demonstrated that this drug significantly, but not completely, reversed the ability of conditioned medium from HRV-infected HBE to inhibit TGF $\beta 1$ to induce a-SMA expression in airway fibroblasts. This implied that, while other mediators likely contribute to the inhibitory response seen, one or more prostaglandins play a significant role in inhibiting the ability of TGF $\beta 1$ to induce a-SMA expression in airway fibroblasts. It has previously been reported that $\mathrm{HTV}$-infection of $\mathrm{HBE}$ can induce the generation of several prostaglandins, including $\mathrm{PGE}_{2}$ [25]. Because $\mathrm{PGE}_{2}$ has previously been reported to inhibit TGF $\beta$-induced a-SMA expression [24, 26], we tested the hypothesis that HRV infection induced production of from HBE and that this contributed to inhibition of TGF $\beta 1$ induced a-SMA expression in human airway fibroblasts. Our data clearly demonstrated that $\mathrm{HRV}$ infection of $\mathrm{HBE}$ induced release of $\mathrm{PGE}_{2}$ at levels similar to those reported previously [25]. We also confirmed that $\mathrm{PGE}_{2}$ inhibited TGF $\beta 1$ induced a-SMA expression in a concentration dependent manner. Although mRNA analysis showed that fibroblasts significant expressed mRNA for both $\mathrm{EP}_{2}$ and $\mathrm{EP}_{4}$ receptors for $\mathrm{PGE}_{2}$, data using both selective antagonists and agonists demonstrated that effects of $\mathrm{PGE}_{2}$ were mediated entirely by interactions at the $\mathrm{EP}_{2}$ receptor, a G-protein coupled receptor with a Gsa subunit that activated adenylyl cyclase to increase intracellular levels of cAMP [27].

It is apparent that the interaction of HRV-infected airway epithelial cells with airway fibroblasts is complex. Although our current data show that supernatants from infected epithelial cells can reduce FMT, HRV-infected cells also release chemokines that are chemotactic for fibroblasts and could contribute to increased fibroblast numbers in the region of the lamina reticularis [17]. Such fibroblasts also secrete matrix proteins that could lead to enhanced matrix protein deposition. Moreover, infected epithelial cells secrete a number of growth factors that can stimulate matrix protein deposition from fibroblasts and myofibroblasts [16].

A limitation of our study is that we used only epithelial cells and fibroblasts from healthy normal controls and, as such, our data may not fully represent data that may be obtained with cells derived from asthmatic airways. It is known that airway epithelial cells phenotype is altered in both children and adults with asthma [28, 29]. Moreover, several phenotypic changes in epithelial cells from asthmatic subjects, including increased expression of cytokeratin 5, altered production of cytokines and chemokines, and enhanced production of several growth factors linked to airway remodeling are maintained in culture [28, $30-33]$. Thus, given that airway remodeling occurs in patients with asthma but not in healthy normal subjects, it would seem likely that epithelial cells from asthmatic subjects may exert properties that would be more supportive of aspects of remodeling. In support of this concept, it has been reported that coculture of epithelial cells from asthmatic subjects with fibroblasts in the presence of TGF $\beta$ enhances expression compared to cells from normal subjects [26]. Interestingly, it has been reported that, while expression of TGF $\beta$ is increased upon co-culture of fibroblasts with asthmatic epithelial cells, expression of prostaglandin $E_{2}$ synthase is downregulated compared to co-cultures with normal epithelial cells [33]. Thus, one may speculate that enhanced $\mathrm{PGE}_{2}$ expression in normal cells, along with reduced expression may help to prevent airway remodeling in normal subjects. Further studies will be required to address this. 
Finally, it must be noted that we are unaware of any studies thus far in which the interaction of asthmatic airway epithelial cells with fibroblasts also derived from asthmatic patients have been examined. It is feasible that this such studies may further exaggerate differences between normal and asthmatic cell models.

In summary, our data demonstrate that HRV-induced production of $\mathrm{PGE}_{2}$ from airway epithelial cells derived from normal subjects inhibits TGF $\beta$-induced FMT. We speculate that this may contribute to preventing airway remodeling in normal subjects.

\section{Declarations}

\section{Ethics Approval:}

All research experiments using cells obtained from human subjects were performed in accordance with the Declaration of Helsinki, and ethical approval to obtain cells from normal human lungs was obtained from both the Conjoint Health Research Ethics Board of the University of Calgary and the Internal Ethics Board of the International Institute for the Advancement of Medicine (Edison, $\mathrm{NJ}$ ), the tissue retrieval service used to obtain lungs not used for transplant. Informed consent was obtained from next of kin of each donor.

\section{Consent for Publication:}

Not applicable.

\section{Availability of Data:}

All data from this study are available in the published article and in the Supplemental Information file.

\section{Competing Interests:}

The authors declare that they have no competing interests.

\section{Funding:}

This work was supported by the Canadian Institutes of Health Research (Project grant PJT-159635). DMP was supported by a scholarships from the Lung Association of Alberta and NWT, and the GoranEnhorning Scholarship from Asthma Canada-Allergen NCE. DP holds a Tier 1 Canada Research Chair in Inflammatory Lung Diseases. RL holds the GlaxoSmithKline-CIHR Professorship in Inflammatory Lung Diseases.

\section{Authors Contributions:}

DMP, DP and RL conceived and planned the experimental design. DMP performed experiments and analyzed data. CK derived and propagated the fibroblasts used in this study, provided technical support 
for all aspects of the study, and assisted in generating the data for Figure 1. All authors contributed to the writing and editing of the manuscript and all authors approved the final manuscript.

\section{Acknowledgements:}

The authors gratefully acknowledge assistance from Shahina Wiehler in deriving epithelial cells and virus preparations.

\section{References}

1. Al-Muhsen S, Johnson JR, Hamid Q: Remodeling in asthma. J Allergy Clin Immunol 2011, 128:451462.

2. Hough KP, Curtiss ML, Blain TJ, Liu R-M, Trevor J, Deshane JS, Thannickal VJ: Airway remodeling in asthma. Front Med 2020, 7:191.

3. Ingram JL, Huggins MJ, Church TD, Li Y, Francisco DC, Degan S, Firszt R, Beaver DM, Lugogo NL, Wang Y, et al: Airway fibroblasts in asthma manifest and invasive phenotype. Am J Respir Crit Care Med 2011, 183:1625-1632.

4. Powell DW, Mifflin RC, Velentich JD, Crowe SE, Saada JI, West JB: Myofibroblasts. I. Paracrine cells important in health and disease. Am J Physiol 1999, 277:C1-C9.

5. Hinz B, Phan SH, Thannickal VJ, Prunotto M, Desmouliere A, Varga J, De Wever O, Mareel M, Gabbiani G: Recent developments in myofibroblast biology: paradigms for connective tissue remodeling. Am J Pathol 2012, 180:1340-1355.

6. Eyden B: The myofibroblast: phenotypic characterization as a prerequisite to understanding its function in translational medicine. J Cell Mol Med 2008, 12:22-37.

7. SIngh SR, Hall IP: Airway myofibroblasts and their relationship with airway myocytes and fibroblasts. Proc Am Thorac Soc 2008, 5:127-132.

8. Michalik M, Wójcik K, Paw M, Wnuk D, Koczurkiewicz P, Sanak M, Pekala E, Madeja Z: Fibroblast-tomyofibroblast transition in bronchial asthma. Cell Mol Life Sci 2018, 75:3943-3961.

9. Weitoft M, Andersson C, Andersson-Sjöland A, Tufvesson E, Bjermer L, Erjefalt J, WestergrenThorsson G: Controlled and uncontrolled asthma display distinct alveolar tissue matrix compositions. Respir Res 2014, 15:67.

10. Pepe C, Foley S, Shannon J, Lemiere C, Olivenstein R, Ernst P, Ludwig M, Martin J, G., Hamid Q: Differences in airway remodeling between subjects with severe and moderate asthma. J Allergy Clin Immunol 2005, 116:544-549.

11. Schmidt M, Sun G, Stacey MA, Mori L, Mattoli S: Identification of circulating fibrocytes as precursors of bronchial myofibroblasts in asthma. J Immunol 2003, 171:380-389.

12. McAnulty RJ: Fibroblasts and myofibroblasts: their source, function and role in disease. Int $J$ Biochem Cell Biol 2007, 39:666-671. 
13. Falke LL, Gholizadeh S, Goldschmeding R, Kok RJ, Nguyen TQ: Diverse origins of myofibroblastsimplications for kidney fibrosis. Nat Rev Nephrol 2015, 11:233-244.

14. Redington AE, Madden J, Frew AJ, Djukanovic R, Roche W, Holgate ST, Howarth PH: Transforming growth factor- $\beta 1$ in asthma. Measurement in bronchoalveolar lavage fluid. Am J Respir Crit Care Med 1997, 156:642-647.

15. Michalik M, Pierzchalska M, Legutko A, Ura M, Ostaszexska A, Soja J, Sanak M: Asthmatic bronchial fibroblasts demonstrate enhanced potential to differentiate into myofibroblasts in culture. Med Sci Monit 2009, 15:BR194-201.

16. Leigh R, Oyelusi W, Wiehler S, Koetzler R, Zaheer RS, Newton R, Proud D: Human rhinovirus infection enhances airway epithelial cell production of growth factors involved in airway remodeling. $J$ Allergy Clin Immunol 2008, 121:1238-1245.

17. Shelfoon C, Shariff S, Traves SL, Kooi C, Leigh R, Proud D: Chemokine release from human rhinovirus-infected airway epithelial cells promotes fibroblast migration. J Allergy Clin Immunol 2016, 138:110-122.

18. Zhu L, Lee PK, Lee W-M, Zhao Y, Yu D, Chen Y: Rhinovirus-induced major airway mucin production involves a novel TLR3-EGFR-dependent pathway. Am J Respir Cell Mol Biol 2009, 40:610-619.

19. Tacon CE, Wiehler S, Holden NS, Newton R, Proud D, Leigh R: Human rhinovirus infection of airway epithelial cells upregulates MMP-9 production via NF-KB. Am J Respir Cell Mol Biol 2010, 43:201-209.

20. Minor DM, Proud D: Role of human rhinovirus in triggering human airway epithelial-mesenchymal transition. Respir Res 2017, 18:110.

21. Sanders SP, Siekierski ES, Porter JD, Richards SM, Proud D: Nitric oxide inhibits rhinovirus-induced cytokine production and viral replication in a human respiratory epithelial cell line. J Virol 1998, 72:934-942.

22. Subauste MC, Jacoby DB, Richards SM, Proud D: Infection of a human respiratory epithelial cell line with rhinovirus. Induction of cytokine release and modulation of susceptibility to infection by cytokine exposure. J Clin Invest 1995, 96:549-557.

23. Claar D, Hartert TV, Peebles RS: The role of prostaglandins in allergic lung inflammation and asthma. Expert Rev Respir Med 2015, 9:55-72.

24. Penke LRK, Huang SK, White ES, Peters-Golden M: Prostaglandin $E_{2}$ inhibits -smooth muscle actin transcription during myofibroblast differentiation via distinct mechanisms of modulation of serum response factor and myocardin-related transcription factor-A. J Biol Chem 2014, 289:17151-17162.

25. Van Ly D, Faiz A, Jenkins C, Crossett B, Black JL, McPartland B, Burgess JK, Oliver BGG: Characterising the mechanism of airway smooth muscle $\beta 2$ adrenoreceptor desensitization by rhinovirus infected bronchial epithelial cells. PLoS One 2013, 8:e56058.

26. Reeves SR, Kolstad T, Lien T-Y, Herrington-Shaner S, Debley JS: Fibroblast-myofibroblast transition is differentially regulated by bronchial epithelial cells from asthmatic children. Respir Res 2015, 16:21. 
27. Narumiya S: Prostanoid receptors. Structure, function, and distribution. Ann N Y Acad Sci 1994, 744:126-138.

28. Carsin A, Mazenq J, Ilstad A, Dubus J-C, Chanez P, Gras D: Bronchial epithelium in children: a key player in asthma. Eur Respir Rev 2016, 25:158-169.

29. Loxham M, Davies DE, Blume C: Epithelial function and dysfunction in asthma. Clin Exp Allergy 2014, 44:1299-1313.

30. Hackett T-L, Singhera GK, Shaheen F, Hayden P, Jackson GR, Hegele RG, Van Eeden S, Bai TR, Dorscheid DR, Knight DA: Intrinsic phenotypic differences of asthmatic epithelium and its inflammatory responses to respiratory syncytial virus and air pollution. Am J Respir Cell Mol Biol 2011, 45:1090-1100.

31. Kicic A, Sutanto EN, Stevens PT, Knight DA, Stick SM: Intrinsic biochemical and functional differences in bronchial epithelial cells of children with asthma. Am J Respir Crit Care Med 2006, 174:1110-1118.

32. Lopez-Guisa JM, Powers C, File D, Cochrane E, Jiminez N, Debley JS: Airway epithelial cells from asthmatic children differentially express proremodeling factors. J Allergy Clin Immunol 2012, 129:990-997.

33. Reeves SR, Kolstad T, Lien Y-U, Elliott M, Ziegler SF, Wight TN, Debley JS: Asthmatic airway epithelial cells differentially regulate fibroblast expression of extracellular matrix components. J Allergy Clin Immunol 2014, 134:663-670.

\section{Figures}


c.

A.

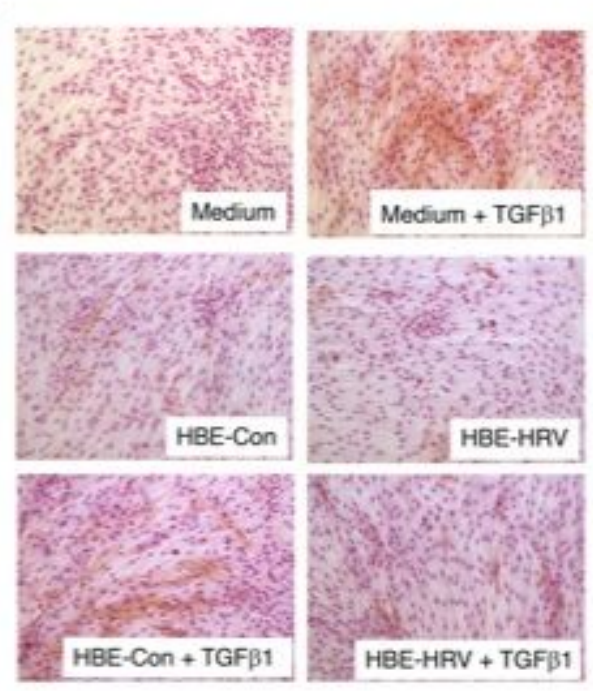

B.

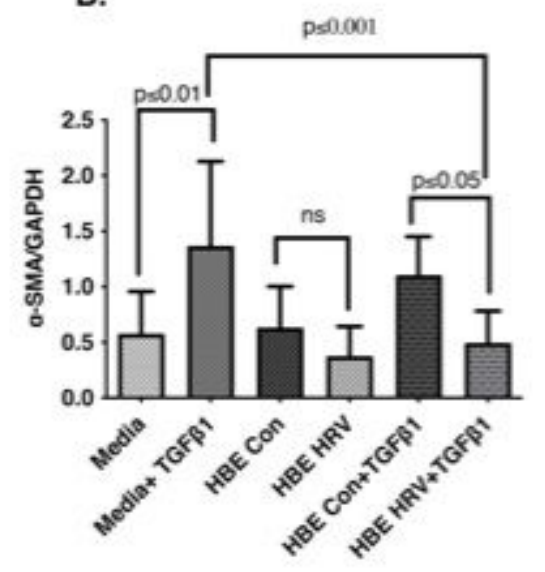

D.

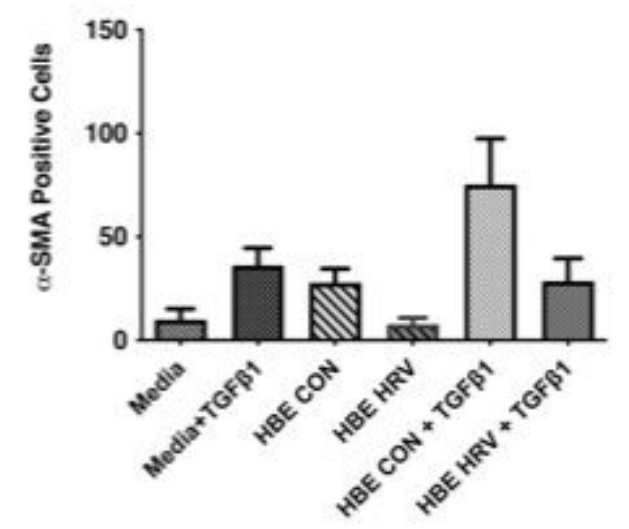

Figure 1

HRV-16 conditioned supernatant modulated TGF $\beta 1$-induced a-SMA expression in human bronchial fibroblasts.

A. Representative western blot analysis of a-SMA in a HBF donor stimulated with medium alone, $1 \mathrm{ng} / \mathrm{ml}$ TGF 31 alone, conditioned medium from non-infected epithelial cells (HBE-Con), conditioned medium from HRV-16 infected epithelial cells (HBE-HRV), HBE-Con in combination with $1 \mathrm{ng} / \mathrm{ml}$ TGF $\beta 1$ and HBEHRV in combination with $1 \mathrm{ng} / \mathrm{ml}$ of TGF $\beta 1$. B. Densitometric analysis of a-SMA protein expression normalized over GAPDH protein expression in from 8 different fibroblast donors. Data were analyzed by one-way ANOVA with post-hoc test Bonferroni's multiple comparisons test. Data are expressed as mean \pm SEM (ns = not significant). C. Representative immunohistochemical analysis at 100X magnification of aSMA positive cells after $48 \mathrm{~h}$ of stimulations. Brown stains represent the location and presence of a-SMA. Data were expressed as mean \pm SEM and are representative of $n=3$. D. Densitometric analysis of immunohistochemical data from the 3 donors. 
A.

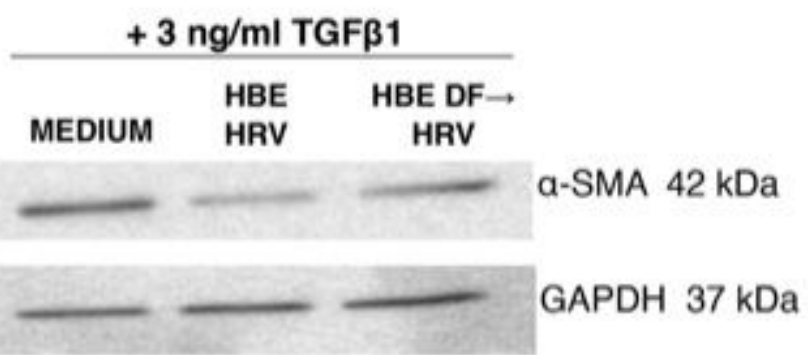

B.

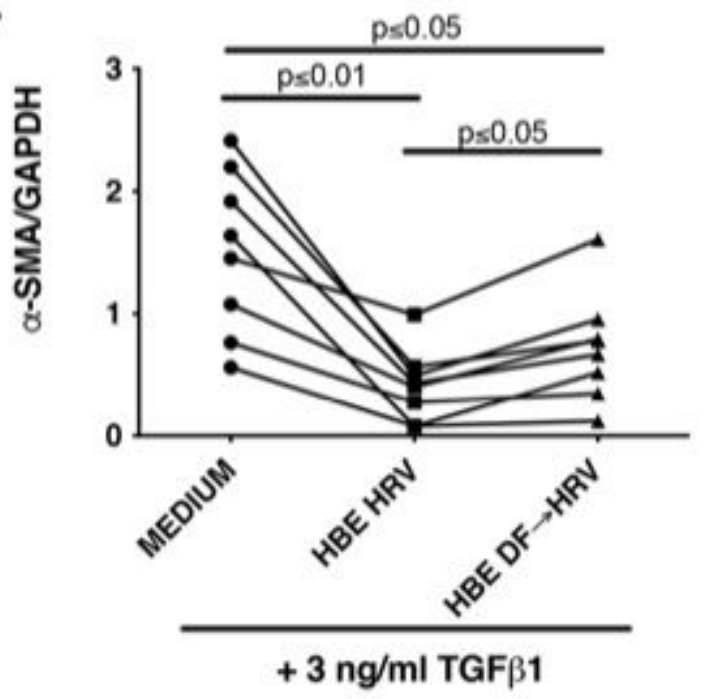

Figure 2

Diclofenac reverses HBE-HRV mediated suppression of TGF $\beta$ stimulated a-SMA expression in HBFs.

A. Representative western blot analysis of a-SMA in HBFs exposed to TGF $\beta 1$ combined with conditioned medium from HRV-infected HRV stimulated in the presence or absence of diclofenac. B. Mean densitometric analysis of a-SMA normalized over GAPDH expression in HBFs from all experiments. Data are expressed as mean \pm SEM $(n=8)$. Parametric data were statistically analyzed using a one way-ANOVA with appropriate post-hoc testing using the Holm-Sidak multiple comparison test. 


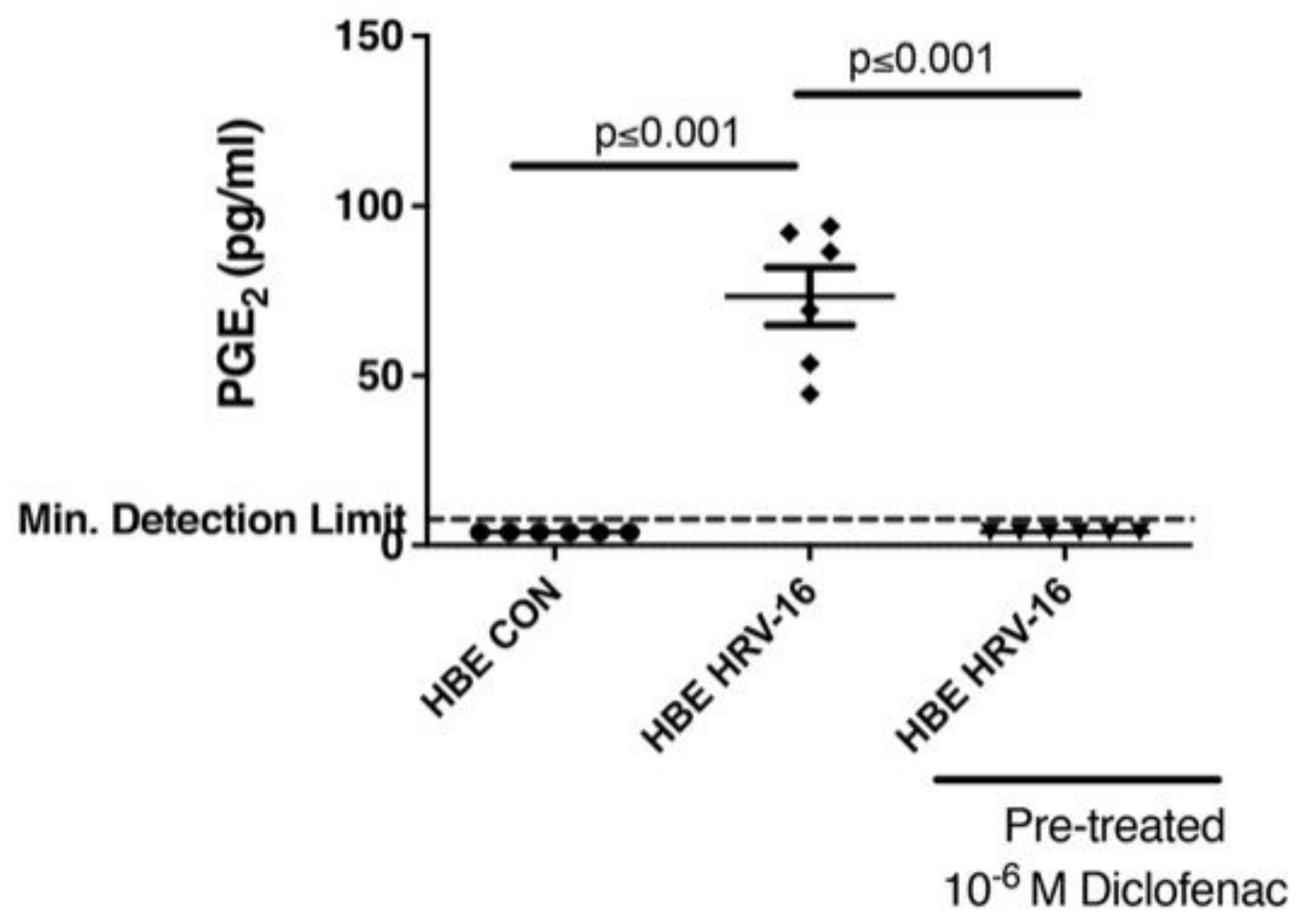

Figure 3

ELISA analysis of prostaglandin $E_{2}$ level in supernatants from human bronchial epithelial cells after $24 \mathrm{~h}$ treatments.

Prostaglandin $\mathrm{E}_{2}$ levels detected in conditioned medium from control cells (HBE-Con) or HBE infected with HRV alone (HBE-HRV) or in the presence of diclofenac. Data were analyzed by one-way ANOVA followed by Bonferroni's multiple comparisons test. Data are expressed as mean \pm SEM $(n=6)$. Values under the minimum detection limit $(7.8 \mathrm{pg} / \mathrm{ml})$ were arbitrarily assigned a value of $3.9 \mathrm{pg} / \mathrm{ml}$. 
A.

$+3 \mathrm{ng} / \mathrm{ml} \mathrm{TGF} \beta 1$

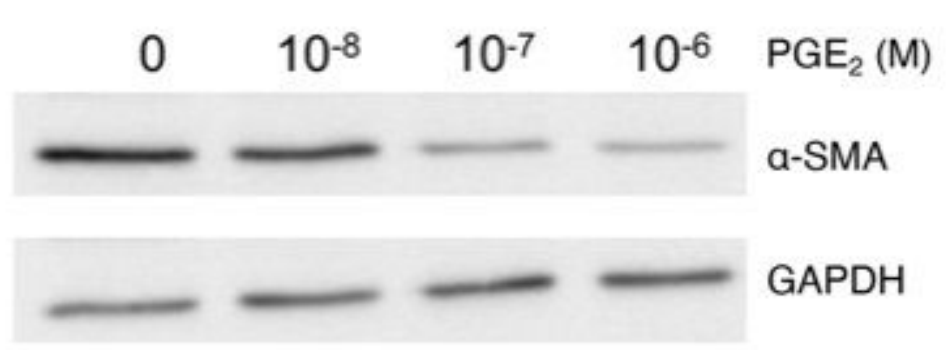

B.

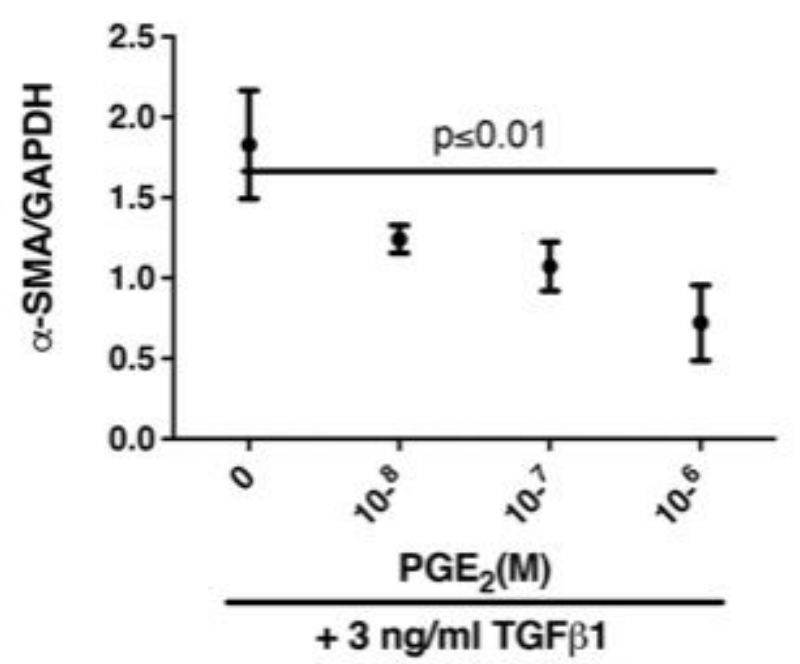

Figure 4

$\mathrm{PGE}_{2}$ significantly inhibits TGF $\beta$ 1-induced a-SMA expression in HBFs.

A. Representative western blot analysis of a-SMA and GAPDH in HBFs after exposure to TGF $\beta 1$ in the presence or absence of varying concentrations of $\mathrm{PGE}_{2}$. B. Densitometric analysis of a-SMA normalized over GAPDH expression in all experiments $(n=5)$. Data are expressed as mean \pm SEM and were analyzed using one way-ANOVA with post-hoc testing comparing each value to the control using Dunnett's multiple comparison test. 
A.

$+3 \mathrm{ng} / \mathrm{ml}$ TGF $\beta 1$
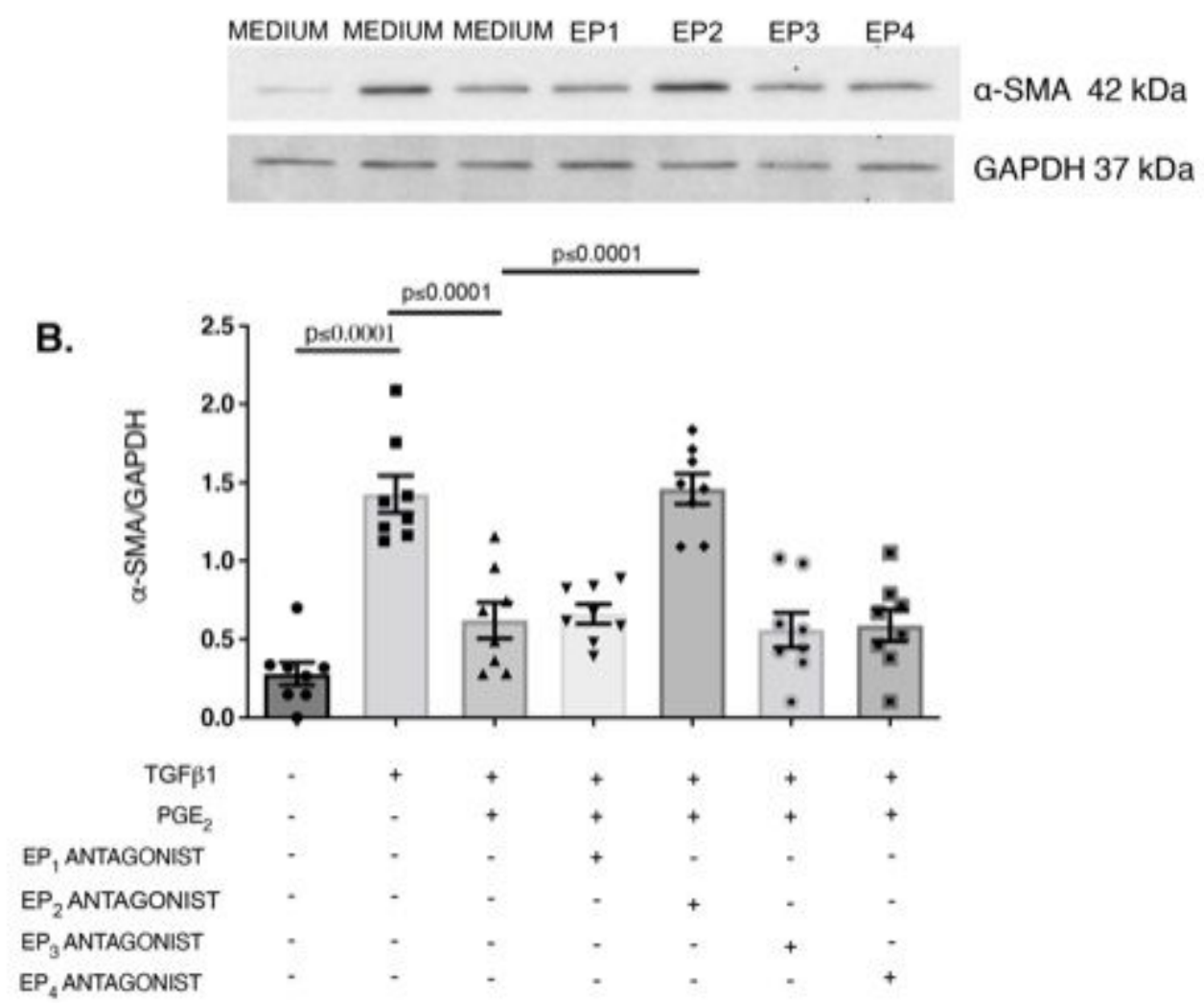

Figure 5

Effects of selective EP receptor antagonists on the inhibition by $\mathrm{PGE}_{2}$ of TGF $\beta 1$-induced $\mathrm{a}-\mathrm{SMA}$ expression.
A. Representative western blot of a-SMA and GAPDH in HBFs exposed to: 1) medium alone, 2) TGF $\beta 1$ alone, 3) TGF $\beta 1$ and $\left.P G E_{2}, 4\right) T G F \beta 1$ and $\mathrm{PGE}_{2}$ in the presence of $\mathrm{EP}_{1}$ antagonist (SC51322), 5) TGF $\beta 1$ and $P G E_{2}$ in the presence of $\mathrm{EP}_{2}$ antagonist (TG4-155), 6) TGF 1 and $\mathrm{PGE}_{2}$ in the presence of $\mathrm{EP}_{3}$ antagonist (L798106), 7) TGF 31 and $\mathrm{PGE}_{2}$ in the presence of $\mathrm{EP}_{4}$ antagonist (L161982). followed by $\mathrm{EP}_{4}$ antagonist, TGF $\beta 1$ and $\mathrm{PGE}_{2}$. All EP antagonists were used at a concentration of $100 \mathrm{nM}$. B.
Densitometric analysis of a-SMA normalized over GAPDH expression for all experiments $(n=8)$. Data were analyzed using one-way ANOVA and post-hoc test Bonferroni's multiple comparisons test. Data are expressed as mean \pm SEM. 
A.
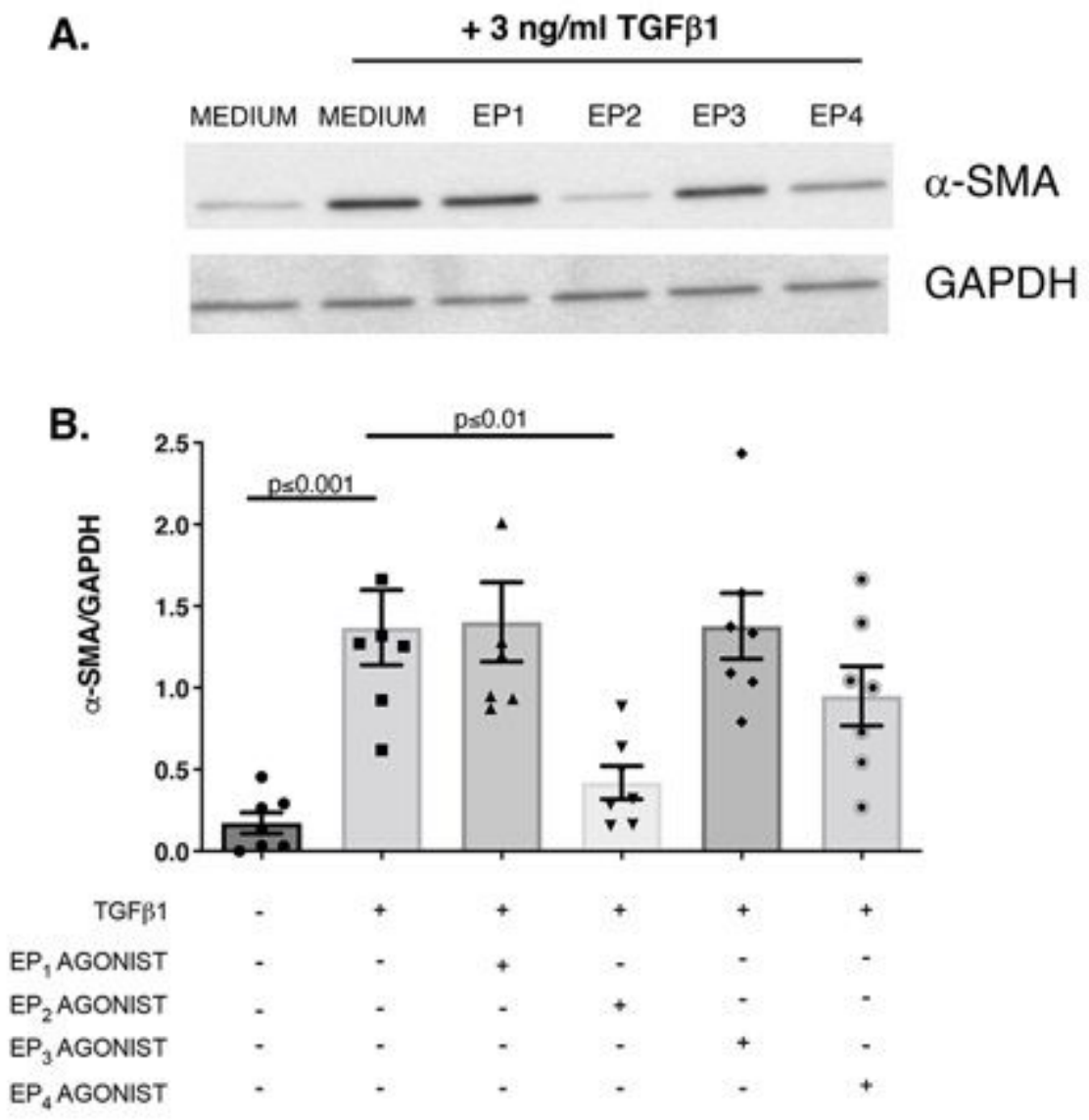

Figure 6

\section{Effects of selective EP receptor agonists on TGFB1-induced a-SMA expression.}

A. Representative western blot of a-SMA and GAPDH in HBFs exposed to: 1) medium alone, 2) TGF 1. alone, 3) TGF $\beta 1$ and $E P_{1}$ agonist (ONO-D1-004), 4) TGF $\beta 1$ and $\mathrm{EP}_{2}$ agonist (ONO-AE1-259-01), 5) TGF 1 and $\mathrm{EP}_{3}$ agonist (ONO-AE-248), and 6) TGFB1 and $\mathrm{EP}_{4}$ agonist (ONO-AE1-329). All EP agonists were used at a concentration of $1 \mu \mathrm{M}$. B. Densitometric analysis of a-SMA normalized over GAPDH expression for all experiments $(n=7)$. Data were analyzed using one-way ANOVA and post-hoc test Bonferroni's multiple comparisons test. Data are expressed as mean \pm SEM.

\section{Supplementary Files}

This is a list of supplementary files associated with this preprint. Click to download.

- SupplementaryInformation.docx 